\title{
Étude descriptive de l'utilisation des cartes conceptuelles comme stratégie pédagogique en sciences de la santé
}

\section{A descriptive study of concept maps use as pedagogical strategy in health sciences}

\author{
Annie RocheTte ${ }^{1,2}$, Marilou BÉLISLE ${ }^{3}$, André LAFLAMME ${ }^{4}$, Michèle DouceT ${ }^{5}$, \\ Monique CHAPUT $^{6}$ et Barbara FILLION ${ }^{2}$
}

1 Programme d'ergothérapie, École de réadaptation, Faculté de médecine, Université de Montréal, Montréal, Québec, Canada

2 Centre de recherche interdisciplinaire en réadaptation du Montréal Métropolitain (CRIR), Montréal, Québec, Canada

3 Centre d'études et de formation en enseignement supérieur (CEFES), Université de Montréal, Montréal, Québec, Canada

4 Bureau de l'environnement numérique d'apprentissage (BENA), Université de Montréal, Montréal, Québec, Canada

5 Faculté de médecine vétérinaire, Université de Montréal, St. Hyacinthe, Montréal, Québec, Canada

6 Centre de pédagogie appliquée aux sciences de la santé, Faculté de médecine, Université de Montréal, Montréal, Québec, Canada

Manuscrit reçu le 21 janvier 2010 ; commentaires éditoriaux formulés aux auteurs le 16 mai 2010 ; accepté pour publication le 21 juillet 2010
Mots clés :
Cartes conceptuelles ;
pratiques actuelles ;
sciences de la santé ;
intégration
des connaissances ;
évaluation
des connaissances
Résumé - Contexte : Les cartes conceptuelles peuvent répondre à des usages multiples en éducation en sciences de la santé, comme stratégie de communication, comme aide à l'ap- prentissage, comme aide à la planification ou comme soutien à l'évaluation. Mais leur uti- lisation réelle dans le champ de la formation en sciences de la santé est mal connue. But : Les buts de cette étude exploratoire étaient : de documenter les pratiques actuelles d'utilisa- tion des cartes conceptuelles dans le quotidien des professeurs en sciences de la santé ayant suivi une formation en ce domaine; d'identifier les conditions perçues comme favorisant ou freinant leur utilisation. Méthodes : La population visée était les professeurs en sciences de la santé ayant suivi une formation sur les cartes conceptuelles dans deux universités qué- bécoises $(n=55)$. Un questionnaire en ligne a été conçu spécifiquement pour cette étude exploratoire. Une analyse comparative du contenu permettant de faire ressortir les simili- tudes et différences a été réalisée. Résultats : Plus de trois quart des répondants ( $n=26 / 33)$ rapportent utiliser les cartes conceptuelles. Leur utilisation est très variée : simple ou com- plexe, en prestation d'enseignement ou en évaluation, carte créée par le professeur et/ou les étudiants, construite en mode individuel ou collaboratif, en petit ou grand groupe. L'appren- tissage signifiant est identifié comme un avantage important des cartes conceptuelles alors que le temps supplémentaire requis est perçu comme un des obstacles à leur utilisation. Conclu- sion : Les pratiques actuelles d'utilisation des cartes conceptuelles en éducation en sciences de la santé sont diversifiées et les obstacles identifiés apparaissent surmontables, ce qui est prometteur pour l'avenir. 


\section{Keywords:} Concept map; actual practices; heath sciences; knowledge integration; knowledge assessment
Abstract - Context: Concept maps are useful in a variety of ways such as communication strategies, learning tools, planning tools or assessments. Yet what is known about their current use in health sciences? Purpose: Describing current concept map practices by health sciences professors who benefited from specific training and secondly identifying perceived facilitators and hurdles to their use. Methods: The target group included all health science professors who were trained in concept mapping in two Quebec's universities $(n=55)$. Data were collected using an exploratory on line questionnaire developed specifically for this study. A comparative analysis of the content helped identify similarities and differences. Results: More than three quarters of respondents $(n=26 / 33)$ reported using concept maps in one or several courses. Their use varied greatly, from simple to complex, in individual or collaborative mode, in small or large size groups, from teaching strategies to assessments and maps created by teachers or students. Meaningful learning came out as an advantage while the extra time needed proved to be a hurdle. Conclusion: Current concept map practices in health sciences are varied. Hurdles could be overcome, which is promising for the future.

\section{Introduction}

Les cartes conceptuelles peuvent répondre à des usages multiples en éducation en sciences de la santé, comme stratégie de communication, aide à l'apprentissage, comme aide à la planification ou comme soutien à l'évaluation. Mais leur utilisation réelle dans le champ de la formation en sciences de la santé est mal connue. Nous présentons une étude exploratoire visant à documenter les pratiques actuelles d'utilisation des cartes conceptuelles dans le quotidien des professeurs en sciences de la santé ayant suivi une formation en ce domaine. Un second objectif consiste à identifier les conditions perçues comme favorisant ou freinant à leur utilisation.

\section{Problématique}

Utilité des cartes conceptuelles dans une logique d'approche par compétences

Avec l'arrivée de profils de pratique comme CanMeds $^{[1]}$ ou d'autres ${ }^{[2]}$, de plus en plus de programmes de formation se tournent vers une approche par compétences. Par exemple, à l'Université de Montréal, cette approche est actuellement en phase d'implantation dans plusieurs programmes de formation en santé (ergothérapie, médecine, pharmacie, soins infirmiers pour n'en citer que quelques-uns). La compétence se définit comme un
« savoir-agir complexe prenant appui sur la mobilisation et la combinaison efficaces d'une variété de ressources internes et externes à l'intérieur d'une famille de situations ${ }^{[3]}$. La notion d'efficacité est d'autant plus importante que le professionnel de la santé, actuel et en devenir - c.-à-d. l'étudiant -, doit faire face à une augmentation exponentielle des connaissances à maîtriser, ce qui complique le défi d'être en mesure, le moment venu, de les mobiliser et de les combiner efficacement. Dès lors, on peut raisonnablement faire l'hypothèse qu'un travail portant sur l'organisation des connaissances antérieures et des connaissances nouvellement acquises, au moment de l'apprentissage, serait une stratégie cognitive primordiale dans l'optique de la construction d'un savoir-agir ${ }^{[4]}$; il doit donc être encouragé et assisté par les enseignants au cours de la formation. Pour répondre à ce défi, de nouvelles stratégies pédagogiques doivent être adoptées. La carte conceptuelle, avec ses différentes applications ${ }^{[5]}$, pourrait faciliter cette organisation et, à ce titre, elle constitue une technique pédagogique prometteuse dans une logique d'approche par compétences.

Cette étude s'inscrit donc dans cette mouvance vers une approche par compétences de plusieurs programmes de formation professionnelle en santé où de nouvelles stratégies pédagogiques, comme l'utilisation des cartes conceptuelles, sont mises en exergue. 


\section{Qu'est-ce qu'une carte conceptuelle?}

Les cartes conceptuelles prennent appui sur la théorie de l'apprentissage signifiant proposé par David Ausubel ${ }^{[6]}$. Elles permettent un apprentissage «signifiant » (meaningful learning) en favorisant les liens entre les connaissances antérieures et les nouvelles connaissances acquises en cours de formation, comparativement à un apprentissage "par cœur» (rote learning) ${ }^{[7]}$. En tant que tel, leur intégration dans une pédagogie traditionnelle, comme l'enseignement magistral, ne va pas nécessairement de soi $^{[8]}$. Elle prend par contre tout son sens dans le cadre d'une pédagogie active, visant à créer les conditions favorables à la mobilisation de ressources internes (connaissances, habiletés, expériences et attitudes) et externes (documents, outils informatiques, moyens audiovisuels, collègues experts, etc.) par l'étudiant, en cohérence avec les principes directeurs d'une approche par compétences.

Concrètement, la carte conceptuelle a été proposée vers le milieu des années 1980 par Novak. Elle peut se définir comme un «dispositif schématique permettant une représentation visuelle signifiante d'un groupe de concepts, intégrée à des propositions structurées (traduction libre) » ${ }^{[9]}$. Elle permet ainsi, tant à l'enseignant qu'à l'étudiant, d'expliciter les liens entre les différents concepts dont la maîtrise est nécessaire dans le cadre de la construction d'une compétence - tout en signifiant que cette condition n'est pas suffisante, la dimension du savoiragir exigeant davantage que la simple appropriation de concepts). La carte conceptuelle est donc habituellement composée de concepts reliés entre eux par des liens unidirectionnels qui sont nommés (par un verbe), formant ainsi des propositions. L'activité de construction d'une carte conceptuelle consiste ainsi en un effort cognitif intentionnel visant à expliciter et à relier entre eux des concepts en les hiérarchisant, à les relier aux connaissances antérieures, cette démarche étant au cœur de l'apprentissage signifiant. La carte conceptuelle se distingue ainsi d'autres modalités de cartographie, telles que la carte - ou réseau - sémantique - semantic network - (liens habituellement bidirectionnels, sans organisation hiérarchique), de la carte heuristique ou mentale - mind map - (concept central avec représentation arborescente des idées), de la carte cognitive (relations causales, sans explicitation des liens) ou encore de la carte de connaissances - knowledge map - (concepts et liens appartenant à une liste préétablie). Bien que cette approche de la représentation figurative des connaissances ait été formalisée depuis plus de vingt ans, la carte conceptuelle a connu un regain de popularité avec les développements technologiques, c'est-à-dire avec l'arrivée de logiciels informatiques permettant d'apporter une simplification dans le traçage et dans la forme. Plusieurs logiciels de construction de cartes conceptuelles sont disponibles gratuitement sur internet dont, notamment, CmapTools ${ }^{\mathrm{MD}}$ (http://cmap. ihmc. us/download/).

\section{Utilisation possible des cartes conceptuelles en sciences de la santé}

En 2004, Marchand et d'Ivernois ${ }^{[7]}$ ont proposé une synthèse des utilisations possibles des cartes conceptuelles en sciences de la santé. Ils décrivent trois utilisations des cartes conceptuelles. Premièrement, la carte conceptuelle peut servir comme aide à l'apprentissage dans le contexte d'une construction par l'étudiant, individuellement ou en groupe, favorisant ainsi un apprentissage signifiant, la résolution de problème et la mémorisation par la mise en réseau des connaissances. La carte conceptuelle peut aussi servir à l'enseignant comme aide à la planification de l'enseignement et à la conception de design pédagogique, particulièrement dans le contexte de l'approche par compétences où l'on cherche à favoriser les liens entre les différents cours ou, d'une façon générale, lorsque l'on souhaite faciliter l'interdisciplinarité et la collaboration interprofessionnelle autour d'une situation complexe. Finalement, la carte conceptuelle peut servir comme stratégie d'évaluation des apprentissages, particulièrement pour l'identification de propositions (combinaison de concepts et de mots de liaison) erronées ou absentes, ce qui implique que l'on puisse comparer 
les cartes des étudiants à une carte de référence, élaborée par un expert ou un groupe de personnes ressources ou encore que l'on utilise une carte «à trous » (concepts et liens manquants que l'étudiant doit compléter). Plus récemment encore, Daley et Torre $^{[5]}$ ont fait un nouvel inventaire, à partir d'une recension des écrits, des différentes fonctions de la carte conceptuelle dans le domaine de l'éducation médicale, qu'ils classent sous quatre rubriques : 1) favoriser l'apprentissage signifiant ; 2) procurer une ressource additionnelle pour l'apprentissage ; 3) permettre aux instructeurs d'offrir une rétroaction aux étudiants et 4) évaluer les apprentissages et la performance. Ces deux typologies des usages de la carte conceptuelle sont très similaires, quoique la vision de Daley et Torre se centre davantage sur le point de vue de l'étudiant et omet les possibilités d'utilisation du point de vue de l'enseignant pour la planification, que mentionnent Marchand et d'Ivernois.

La conception des cartes ainsi que leur contenu (concept central, histoire de cas, etc.) seront influencés par les objectifs d'utilisation visés. Ainsi, pour obtenir un portrait clair de l'utilisation de la carte conceptuelle dans un contexte donné, les objectifs d'utilisation et les raisons justifiant le choix d'utiliser la carte conceptuelle sont des informations essentielles. Par exemple, les cartes conceptuelles peuvent être utilisées pour l'étude d'un cas ou bien pour permettre la synthèse des connaissances dans un domaine précis ou encore pour évaluer des apprentissages. Les cartes conceptuelles peuvent être utilisées soit uniquement par l'enseignant pour organiser le contenu de son cours, soit uniquement par les étudiants ou à la fois par les étudiants et les enseignants dans un même cours ou à l'intérieur d'un curriculum.

Pourquoi se questionner sur les pratiques actuelles?

Depuis quelques années, le Centre d'études et de formation en enseignement supérieur (CEFES) de l'Université de Montréal offre des formations sur les cartes conceptuelles dans deux universités québécoises aux professeurs intéressés. Cependant, comme il ne suffit pas de se former pour intégrer une nouvelle stratégie d'enseignement dans sa pratique quotidienne, il importe de se questionner sur les pratiques actuelles d'utilisation des cartes conceptuelles en sciences de la santé chez les enseignants ayant suivi cette formation. La formation a-t-elle eu un effet sur les stratégies d'enseignement des professeurs, les objectifs associés à l'utilisation des cartes conceptuelles, les types de cartes produites et le contexte de leur utilisation? Ce contexte d'utilisation est aussi potentiellement influencé par certaines conditions du milieu facilitant ou limitant leur utilisation. Ces conditions peuvent appartenir à l'individu (croyances, connaissances, appropriation de la technique, etc.) ou à l'environnement (accès à un logiciel, infrastructure comme le nombre d'étudiants par groupe, soutien pour la formation, présence d'aide pour la correction, temps, etc.). Ainsi, l'identification des obstacles et des avantages liés à l'utilisation des cartes conceptuelles de même que des opportunités (liés à l'environnement) et limites de son usage (perception des retombées et de la valeur ajoutée), selon la perception des utilisateurs, peut servir de base à des pistes d'actions concrètes pour éventuellement mettre en place des conditions facilitant leur utilisation et, ainsi, réduire l'écart entre les pratiques possibles et les pratiques actuelles.

Afin de documenter les pratiques pédagogiques actuelles qui recourent aux cartes conceptuelles, il convient de se questionner sur le lien entre la logique de leur utilisation et l'arrimage avec les autres approches pédagogiques préconisées ${ }^{[8]}$. Le niveau de conviction de l'enseignant quant à la pertinence de cette stratégie pédagogique et son niveau de confiance quant aux conditions permettant son utilisation avec succès sont des éléments potentiellement importants à considérer ${ }^{[10]}$.

L'objectif principal de cette étude exploratoire est de documenter les pratiques actuelles d'utilisation des cartes conceptuelles dans le quotidien des professeurs en sciences de la santé ayant suivi une formation en ce domaine. Un second objectif consiste à identifier les conditions perçues comme favorisant ou nuisant à leur utilisation. 


\section{Méthodes}

\section{Dispositif de l'étude}

Cette étude descriptive exploratoire utilise un questionnaire administré à l'aide de l'outil de sondage en ligne Survey Monkey ${ }^{\mathrm{MD}}$. Cette méthode de collecte de données a été préférée à des entrevues individuelles ou à des groupes de discussion principalement pour des raisons de faisabilité et de pertinence, puisqu'il s'agissait d'une étude exploratoire voulant dresser un portrait des pratiques effectives du plus grand nombre d'enseignants répondant aux critères d'inclusion. Le questionnaire en ligne permet au répondant de compléter le sondage au moment qui lui convient le mieux, sans qu'il soit nécessaire de prendre un rendez-vous préalablement. Les réponses sont transférables directement dans une base de données, minimisant les possibilités d'erreurs liées à l'entrée des données par une tierce personne et minimisant également les coûts. De plus, bien qu'il eût été intéressant de sonder également les étudiants, la présente étude cible les pratiques des enseignants uniquement, également pour des raisons de faisabilité. Une enquête auprès des étudiants pourrait faire l'objet d'une autre étude.

\section{Population visée}

La population visée était composée des enseignants appartenant à une des sept facultés, écoles ou départements des sciences de la santé de l'Université de Montréal ou de l'Université Laval (médecine, médecine dentaire, médecine vétérinaire, pharmacie, sciences infirmières, kinésiologie et optométrie), ayant suivi une formation sur les cartes conceptuelles offerte par le CEFES entre janvier 2005 et juin $2008(n=55)$. La formation, en petit groupe de 10 à 15 participants, s'étalait sur deux demijournées. Le cadre conceptuel servant de base à la formation s'inscrit dans une approche cognitiviste qui met en valeur l'importance de l'apprentissage signifiant. Lors de cette formation, le participant était amené à construire une carte conceptuelle où il devait mettre en exergue ses connaissances antérieures. Il s'agissait ainsi d'une formation en étapes, au cours de laquelle le participant expérimentait les différentes utilités de la carte conceptuelle, depuis une utilisation simple comme stratégie de communication de l'enseignant vers les étudiants, jusqu'à une utilisation plus complexe impliquant une co-construction, comme lors de la collaboration synchrone et asynchrone dans la construction d'une carte conceptuelle consensuelle. Un travail pratique était assorti à cet atelier : à partir d'un thème proposé par les participants, ceux-ci développaient une carte conceptuelle personnelle qui était discutée en groupe à la rencontre suivante. Cependant, au décours de cette deuxième rencontre, aucun suivi n'était effectué de façon systématique mais le formateur se rendait disponible sur demande, au cas où le participant désirait implanter cette stratégie dans son enseignement et souhaitait bénéficier d'un soutien supplémentaire.

\section{Questionnaire}

Un questionnaire a été développé spécifiquement pour l'étude à partir d'une recension des écrits sur le sujet. Il a fait l'objet de discussions de l'équipe de recherche (cinq réunions sur une période d'environ un an), quant à la formulation des questions et l'ordre de ces dernières, tout en se souciant d'arrimer le contenu recherché au cadre conceptuel. La formulation des questions a été prétestée pour leur compréhension auprès d'un répondant. La première partie du questionnaire était composée de questions ouvertes visant à documenter les pratiques d'utilisation des cartes conceptuelles en incluant le rationnel sous-jacent (objectif principal). Par exemple : Utilisez-vous ou avez-vous déjà utilisé les cartes conceptuelles comme stratégie d'enseignement et/ou d'apprentissage? Pouvez-vous décrire l'utilisation que vous faites de la carte conceptuelle dans votre enseignement? (veuillez s.v.p. préciser le but et la démarche utilisée), etc. La deuxième partie était composée de questions ouvertes et fermées visant à documenter le contexte de l'utilisation des cartes 
conceptuelles, en mettant l'accent sur la perception de la valeur ajoutée et des facteurs facilitant ou freinant leur utilisation (objectif secondaire). Par exemple : Selon vous, quels sont les points forts ou les avantages (valeur ajoutée) de l'utilisation de la carte conceptuelle dans votre pratique d'enseignement? Selon vous, quelles sont les limites de l'utilisation de la carte conceptuelle dans le contexte de votre pratique? Quels conseils donneriez-vous à un collègue qui voudrait utiliser les cartes conceptuelles dans son enseignement? Etc.

\section{Collecte de données}

Les données ont été recueillies entre le $1^{\text {er }}$ décembre 2008 et le 30 janvier 2009. Les participants ont été invités à participer à l'étude en remplissant le questionnaire en ligne par un courrier électronique personnalisé. Deux rappels par courrier électronique ont été envoyés.

\section{Analyse des données}

Les données des questions fermées ont été interprétées de façon purement descriptive. Une analyse qualitative comparative du contenu des données des questions ouvertes a été réalisée afin de faire ressortir les similitudes et différences selon les contextes d'utilisation, en ce qui concerne le type de carte, son (ses) auteur(s), sa (ses) finalité(s), etc. Ces données ont été synthétisées sous la forme de deux cartes conceptuelles, co-construites en collaboration par deux membres de l'équipe de recherche pour ensuite faire l'objet de discussions avec les autres membres de l'équipe jusqu'à l'obtention d'un consensus.

\section{Résultats}

Les données des questions fermées sont présentées de façon descriptive dans les tableaux I à III. Les données qualitatives extraites des questions ouvertes sont présentées sous la forme de deux cartes conceptuelles qui font l'objet des figures 1 et 2 .
Tableau I. Caractéristiques des enseignants ayant répondu au questionnaire sur l'utilisation des cartes conceptuelles $(n=33)$.

Genre $\quad$ Fréquence*

Femme 23

Homme

10

$\begin{array}{lc}\text { Groupe d'âge } \\ 20 \text { à } 39 \text { ans } & 4 \\ 40 \text { à } 49 \text { ans } & 15 \\ 50 \text { et plus } & 14\end{array}$

\section{Titre d'emploi}

Professeur 22

Autres (chargé d'enseignement,

responsable formation

clinique ou pratique,

chargé de projet)

$\begin{array}{lr}\text { Expérience en enseignement (années) } \\ <10 \text { ans } & 11 \\ \text { Entre } 10 \text { ans et } 24 \text { ans } & 14 \\ 25 \text { et plus } & 8\end{array}$

\section{Nombre d'étudiants par groupe}

50 et moins 3

Entre 51 et 100 étudiants

Entre 101 et 200 étudiants

Plus de 200 étudiants 7

* Données manquantes pour certains répondants

\section{Description de l'échantillon}

Des 55 personnes sollicitées, 33 ont rempli le questionnaire en ligne, soit un taux de réponse de $60 \%$. Parmi les répondants, huit d'entre eux $(24,2 \%)$ ont rapporté ne pas utiliser les cartes conceptuelles dans leur pratique. Les raisons de non-utilisation évoquées étaient : un manque de temps $(n=3)$; ne s'applique pas à leur contexte $(n=3)$; ne l'ont pas priorisé comme stratégie $(n=1)$ et donnée manquante $(n=1)$. La majorité des répondants enseignaient à des groupes de plus de 51 étudiants $(n=20 / 33)$ et 
Tableau II. Pratiques d'utilisation des cartes conceptuelles en éducation en sciences de la santé déclarées par les répondants au questionnaire de l'étude $(n=26)$.

\begin{tabular}{lc}
\hline & Fréquence* \\
\hline $\begin{array}{l}\text { L'enseignant conçoit la carte }(n=7) \\
\text { Type de carte conceptuelle } * *\end{array}$ & \\
Carte thématique & 4 \\
Carte contextuelle & 2 \\
Autre (les deux) & 1 \\
Méthode de traçage & \\
Papier crayon & 1 \\
Cmap Tools & 6 \\
MOT ou MOT+ & 1
\end{tabular}

$\begin{array}{ll}\begin{array}{l}\text { Les étudiants conçoivent la carte }(n=19) \\ \text { Type de carte conceptuelle** }\end{array} & \\ \text { Carte thématique } & 7 \\ \text { Carte contextuelle } & 5 \\ \text { Autre (les deux) } & 7\end{array}$

\begin{tabular}{|c|c|c|c|}
\hline \multicolumn{4}{|l|}{ Méthode de traçage } \\
\hline \multicolumn{2}{|l|}{ Papier crayon } & 6 & \\
\hline \multicolumn{2}{|l|}{ CmapTools } & 16 & \\
\hline \multicolumn{2}{|l|}{ MOT ou MOT+ } & 1 & \\
\hline \multicolumn{2}{|l|}{ Autre : au choix de l'étudiant } & 1 & \\
\hline \multicolumn{4}{|l|}{$\begin{array}{l}\text { Présentation de l'utilisation } \\
\text { de la carte aux étudiants }\end{array}$} \\
\hline & & 9 & \\
\hline \multicolumn{4}{|l|}{$\begin{array}{l}\text { Oui } \\
\text { Type de présentation }\end{array}$} \\
\hline \multicolumn{2}{|l|}{ Orale } & 7 & \\
\hline \multicolumn{2}{|l|}{ Écrite } & 3 & \\
\hline \multicolumn{2}{|l|}{ Discussion } & 3 & \\
\hline \multicolumn{2}{|l|}{ Autres (APP, dépôt prof, oral ET écrit) } & 3 & \\
\hline \multicolumn{4}{|l|}{ Rétroaction sur la carte conceptuelle? } \\
\hline \multicolumn{2}{|l|}{ Oui } & 16 & \\
\hline \multicolumn{4}{|l|}{ Recueil de l'opinion des étudiants } \\
\hline \multicolumn{2}{|l|}{ Sondage } & 1 & \\
\hline \multicolumn{2}{|l|}{ Discussion structurée } & 2 & \\
\hline \multicolumn{2}{|l|}{ Discussion informelle } & 5 & \\
\hline \multicolumn{2}{|l|}{$\begin{array}{l}\text { Autre (évaluation formelle de fin } \\
\text { de session) }\end{array}$} & 1 & \\
\hline Lieu où la carte est conçue par l'étudiant & Papier & & Logiciel \\
\hline En classe & 6 & & 8 \\
\hline Hors classe & 4 & & 15 \\
\hline
\end{tabular}

* Données manquantes pour certains répondants et possibilités de plusieurs réponses.

** Carte thématique (portant sur un concept ou une question centrale) et carte contextuelle (décrivant les éléments propres à une histoire de cas ou à une vignette). 
les deux tiers ( $n=22 / 33)$ avaient plus de dix années d'expérience en enseignement (voir tableau I). Le dénominateur varie en raison de données manquantes.

\section{Pratiques d'utilisation des cartes conceptuelles}

\section{La carte construite par l'enseignant}

Parmi les 26 répondants utilisant les cartes conceptuelles dans leur pratique, sept rapportaient concevoir eux-mêmes les cartes. Ils rapportaient utiliser la carte conceptuelle pour organiser et structurer un cours ou en résumer son contenu. Cette technique était perçue comme une représentation plus dynamique que statique et facilitant la visualisation du processus, d'un raisonnement ou d'une démarche (voir figure 1). À titre d'exemple, un répondant mentionnait : "Représentation visuelle complète et dynamique d'une situation. Bon soutien pour accompagner une explication ou un contenu; rejoint plus d'étudiants que seulement l'explication verbale ou la présentation linéaire sur PowerPoint ». Cependant, pour un autre répondant, la perception de la valeur ajoutée découlait d'une rétroaction obtenue des étudiants : «Il m'est difficile d'évaluer l'impact chez les étudiants, tant au niveau compréhension, qu'étude mais lors de l'évaluation du cours, je reçois parfois des commentaires à l'effet que c'est apprécié. Pour moi, cela me permet de bien structurer les différentes sections du cours ».

\section{La carte construite par les étudiants}

Pour les autres répondants $(n=19)$, le scénario privilégié consistait à faire construire les cartes conceptuelles par les étudiants, afin de les inciter à faire des liens, à organiser les connaissances, à intégrer les connaissances antérieures aux nouvelles connaissances acquises, etc. Cette utilisation est diversifiée en ce sens qu'elle pouvait se faire dans un contexte individuel ou d'équipe, lors d'une pédagogie traditionnelle (magistrale) ou d'une approche par problèmes et faire l'objet d'une évaluation sommative
Tableau III. Utilisation de la carte conceptuelle comme un soutien à l'évaluation par les répondants au questionnaire.

\begin{tabular}{lcc}
\hline & $\begin{array}{c}\text { Évaluation } \\
\text { formative* } \\
(n=12 / 18)\end{array}$ & $\begin{array}{c}\text { Évaluation } \\
\text { sommative* } \\
(n=5 / 17)\end{array}$ \\
\hline Qui évalue les cartes? & 8 & 4 \\
Professeurs & 1 & 1 \\
Professeur et l'étudiant & 1 & \\
Tuteurs & 1 & 1 \\
Professeurs et tuteurs & 1 & \\
Professeurs et correcteurs & &
\end{tabular}

\section{Comment les cartes \\ sont-elles évaluées?}

Selon un barème 5

Commentaires inscrits

1

sur la carte

Discussion individuelle $\quad 1$

Évaluation écrite $\quad 1$

Discussion informelle $\quad 1$

\section{Existence d'un corrigé ? $\quad 6 / 18$}

* Données manquantes pour certains répondants (les chiffres représentent la fréquence).

ou formative (voir figure 2). Dans près de la moitié des cas, la carte faisait l'objet d'une présentation orale de la part des étudiants. La majorité utilisait le logiciel Cmap Tools (voir tableau II). Le tableau III présente les particularités en lien avec l'utilisation de la carte conceptuelle comme une stratégie d'évaluation des apprentissages. Alors que douze répondants mentionnent utiliser la carte conceptuelle comme une évaluation formative, seuls cinq l'utilisent à titre d'évaluation sommative. Certains utilisent un corrigé «avec les éléments minimaux» mais un autre répondant mentionne : «Il n'y a pas de corrigé pour éviter que les étudiants croient qu'une seule carte est juste... pour éviter aussi que la carte du professeur ne circule par la suite et biaise les résultats des cohortes subséquentes ». 


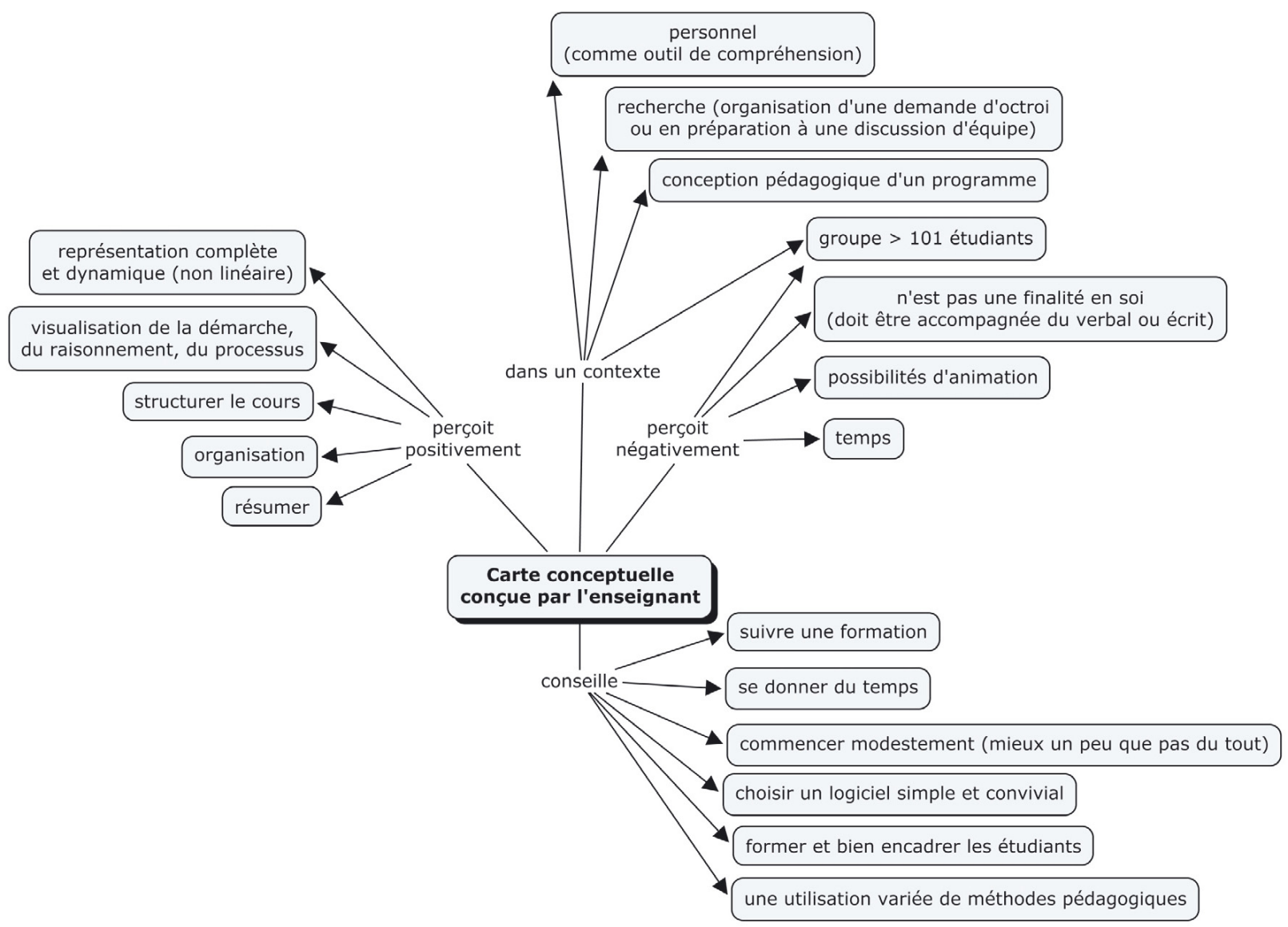

Fig. 1. Carte conceptuelle illustrant la diversité de l'utilisation des cartes conceptuelles par les répondants lorsque cette dernière est construite par l'enseignant.

\section{Obstacles et valeur ajoutée identifiés}

À propos des facteurs identifiés par les répondants comme, respectivement, facilitant ou freinant l'utilisation de cette stratégie dans leur pratique, le temps apparaît être un facteur déterminant. En effet, les répondants recommandaient à un pair entres autres : de suivre une formation, de former adéquatement les étudiants, de se donner du temps pour connaître la technique et de commencer à l'utiliser modestement (voir figures 1 et 2). À titre d'exemple de conseils, un répondant mentionne : «prendre le temps de se former et réserver du temps dans l'horaire pour former les étudiants adéquatement avant de leur demander de créer des cartes »; un autre écrit : «Je n'ai pas vraiment de conseil à donner. Je lui expliquerais le principe et la nécessité d'un entrâेnement qui peut être payant à terme... Mais il faut s'y mettre avec discipline pour atteindre un niveau d'efficacité utile et acquérir le réflexe de l'utiliser», alors que d'autres mentionnent : «GO! GO! GO! Il faut se lancer.. c'est vraiment révélateur de la compréhension des étudiants et si vite à corriger.. on voit tout de suite les erreurs des étudiants » ou encore : «Commencer modestement si on s'adresse à un grand groupe. Il est mieux de l'utiliser un peu que pas du tout».

Lorsque ce sont les étudiants qui construisent la carte conceptuelle, les enseignants identifient également le temps requis pour l'aspect technique comme un facteur important à prendre à compte; en réponse à la question sur les limites, un répondant 


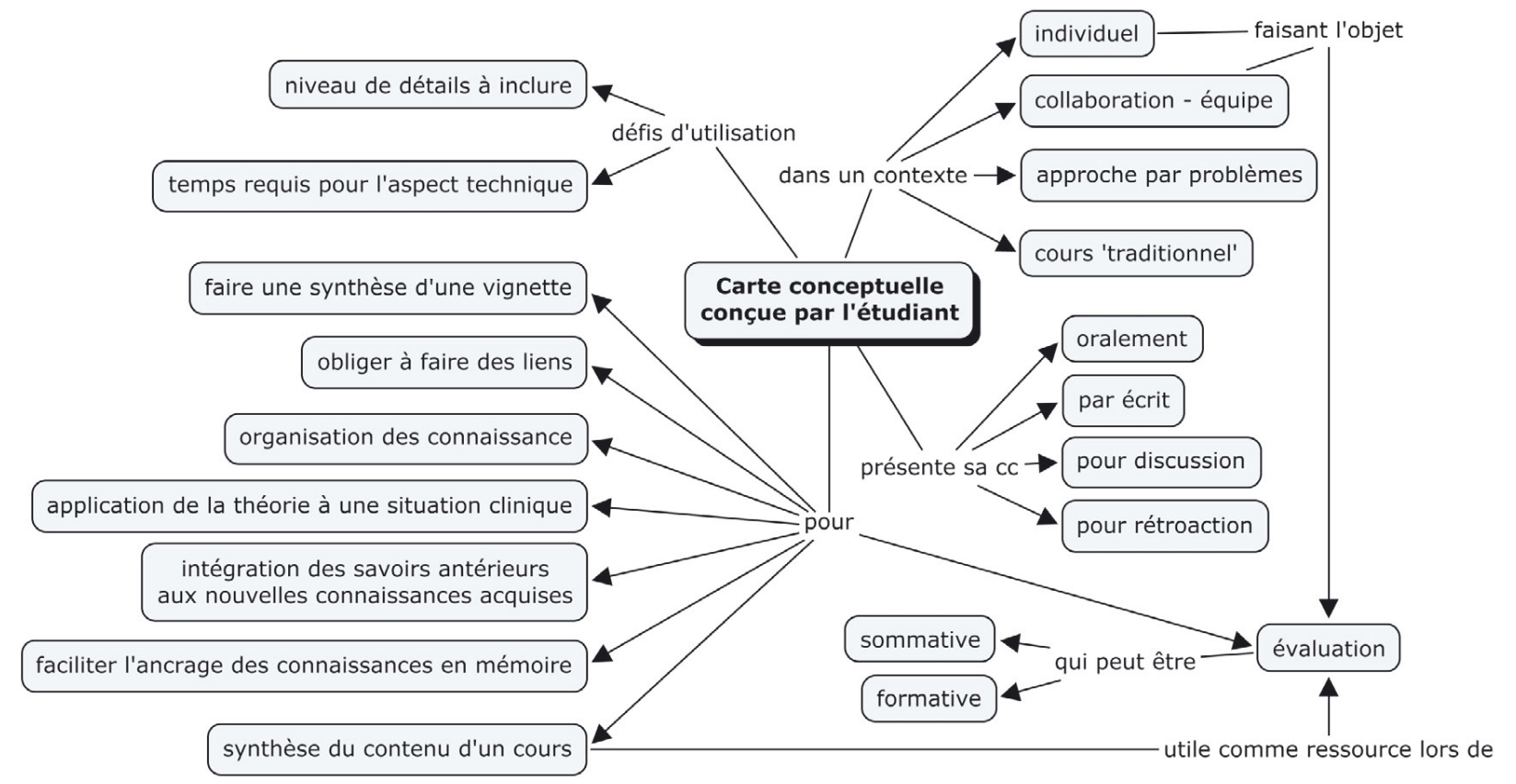

Fig. 2. Carte conceptuelle illustrant la diversité de l'utilisation des cartes conceptuelles par les répondants lorsque cette dernière est construite par l'étudiant.

mentionnait : «Le temps à y consacrer, l'évaluation, la motivation de l'étudiant à utiliser cet outil qui demande effort et énergie. ». Un autre aspect mentionné comme une limite par certains est l'aspect technique associé à l'utilisation d'un logiciel : «Les bornes de la carte demeurent difficiles à définir et suscitent de l'angoisse chez certains étudiants. De l'infiniment grand (explosion de liens, analogue à un discours tangentiel) à l'infiniment petit (décortication obsessive d'un concept), il n'est pas toujours aisé de trouver le juste milieu.» ou : «Les étudiants ont tendance à mettre trop de détails et parfois mettent plus d'énergie sur le design que sur le contenu ».

Parmi les points forts, avantages ou valeurs ajoutées identifiés par les répondants, on y souligne les possibilités de faire une synthèse des connaissances, ce que traduisent par exemple les mentions : «Outil de synthèse facilitant probablement la rétention des points importants. » ou : «pédagogie intégrative et dynamique » ou : «oblige à créer des liens entre les notions apprises; oblige une certaine forme de synthèse; permet la personnalisation des apprentissages. Pour le professeur, permet de voir d'emblée le niveau d'intégration ». On y décrit aussi la possibilité de cerner le niveau de compréhension des étudiants, par exemple en mentionnant : «Permet de faire des liens et de "voir» un peu plus ce qui se passe dans la tête de l'étudiant. Mieux suivre son cheminement.» ou : «Permet de voir la compréhension des étudiants beaucoup plus qu'un examen maison traditionnel... Permet de voir les liens que les étudiants font, ceux qu'ils font mal, ceux qui manquent de nuance, etc. ». Par ailleurs, lorsque la carte conceptuelle est utilisée en mode évaluatif, les critères permettant de l'évaluer correctement ont été rapportés par plusieurs comme un défi d'application.

\section{Discussion}

Cette étude exploratoire est originale et constitue, à notre connaissance, la première visant à recenser les pratiques actuelles d'utilisation des cartes conceptuelles au niveau universitaire en sciences de la santé. Elle comporte néanmoins quelques limites. 
Le taux de non-réponse à certaines questions peut s'expliquer par la méthode utilisée (sondage en ligne). L'utilisation d'une entrevue téléphonique ou en face à face aurait permis de réduire ce nombre de données manquantes. Il est légitime de se questionner sur les caractéristiques des non-répondants taux de réponse de $60 \%$, malgré les relances -; en l'état des informations disponibles, il n'est pas possible de savoir s'ils utilisent dans une proportion équivalente les cartes conceptuelles comme stratégie d'enseignement ou s'ils ont choisi de ne pas répondre au sondage justement parce qu'ils ne les utilisent pas. En ce sens, les résultats de la présente étude exploratoire offrent des pistes quant à la perception des utilisateurs et non-utilisateurs de cette stratégie mais les résultats ne sont pas nécessairement représentatifs de l'ensemble des professeurs en contexte universitaire dans le domaine de la santé, en raison de la petite taille de l'échantillon.

L'objectif principal était de documenter les pratiques actuelles d'utilisation des cartes conceptuelles dans le quotidien des professeurs en sciences de la santé ayant suivi une formation en ce domaine. Les trois utilisations possibles telles que décrites par Marchand et d'Ivernois ${ }^{[7]}$ (aide à l'apprentissage, aide à la planification, aide à l'évaluation) ont été mentionnées. Les résultats indiquent une grande variété d'utilisation qui reflète sans doute la diversité des pratiques d'enseignement rapportées. Cette diversité est telle qu'il a été impossible de définir des profils clairs d'utilisation. Même si les répondants ont tous suivi une même formation, il est possible que les enseignants n'étaient pas tous au même stade d'appropriation de la technique au moment de l'enquête, ce qui pourrait contribuer à expliquer cette grande diversité et l'absence de profil clair, ce qui n'a pu être vérifié statistiquement. Plusieurs répondants utilisant l'approche par problèmes ont rapporté utiliser la carte conceptuelle. Son utilisation est apparue particulièrement utile dans ce contexte tout en ne se limitant pas à cette méthode pédagogique.

Lorsque la carte conceptuelle était une production personnelle demandée aux étudiants, elle faisait l'objet d'une présentation orale auprès d'eux dans près de la moitié des cas. On imagine que suivant cette présentation, une forme de rétroaction était offerte. Selon une étude exploratoire auprès d'étudiants en médecine $(n=134)$, la rétroaction portant à la fois sur la carte en tant que telle (organisation, lisibilité, nombre de détails à inclure, etc.) et sur le contenu (véracité des liens) est essentielle ${ }^{[11]}$. Ceci concorde avec les pratiques actuelles : la majorité des répondants qui font faire la carte par les étudiants l'utilise aussi comme mode d'évaluation (formative), permettant ainsi d'offrir une forme ou une autre de rétroaction. Cependant, quoique l'utilisation d'un barème de correction apparaisse comme une pratique courante, l'existence d'un corrigé est plutôt rare. Les résultats du sondage actuel ne permettent pas d'élucider davantage pourquoi les enseignants ne fournissent pas systématiquement des corrigés aux étudiants alors qu'ils utilisent presque tous un barème de correction pour l'évaluation et/ou la rétroaction. Cela reflète possiblement le désir des enseignants de respecter la nature individuelle et évolutive des cartes conceptuelles, surtout lorsque celles-ci sont produites par les étudiants.

Plus de la moitié des répondants avaient plus de dix ans de carrière en enseignement. Le fait que les enseignants expérimentés pourraient s'intéresser davantage à la pédagogie ou lui accorder une plus grande priorité reste spéculatif. Quoi qu'il en soit, il est légitime de supposer que plus ce type de stratégie est intégré tôt dans la carrière d'un enseignant, plus grand pourrait être l'impact sur sa pratique. On peut en contrepartie faire l'hypothèse qu'une expérience minimale en enseignement soit requise pour que l'utilisation des cartes conceptuelles soit optimale et apporte une réelle valeur ajoutée, comme cela semble être le cas pour les étudiants ${ }^{[12]}$.

Plusieurs logiciels de construction de cartes conceptuelles sont disponibles sur le web, gratuitement ou sous licence, par exemple : Belvedere, SemNet, MOT, Inspiration, PIViT ou Cmap Tools ${ }^{[13]}$. Tous ces logiciels permettent des applications sensiblement similaires. Dans cette étude, les répondants ont certainement été grandement influencés par la 
formation qui utilisait Cmap Tools, puisque la très grande majorité d'entre eux ont rapporté l'utiliser dans leur pratique.

Par ailleurs, près du quart des répondants ont mentionné ne pas utiliser les cartes conceptuelles dans leur pratique, alors même que le fait qu'ils aient suivi une formation sur le sujet démontre un intérêt de leur part à l'égard de cette stratégie. Il est ici intéressant de souligner qu'il ne suffit pas de suivre une formation ou d'avoir un intérêt envers une stratégie pour l'intégrer dans sa pratique. En effet, des efforts importants, associés à un niveau de conviction et de confiance élevé, ainsi qu'à une perception d'une balance avantages/désavantages positive, sont nécessaires pour modifier des habitudes solidement ancrées dans le quotidien et pour intégrer de nouvelles stratégies dans sa pratique ${ }^{[10]}$. Un répondant a suggéré que l'utilisation des cartes conceptuelles devrait être une recommandation explicite et qu'il devrait y avoir une obligation de former les professeurs. Il faudrait alors vérifier que ces derniers intègrent effectivement l'utilisation des cartes conceptuelles dans leurs stratégies d'enseignement, en changeant leur routine de travail.

L'objectif secondaire de l'étude était d'explorer les conditions perçues comme favorisant ou limitant l'utilisation des cartes conceptuelles. Le temps, que ce soit pour l'appropriation de la technique ou la construction d'une carte, est l'obstacle le plus souvent mentionné chez l'ensemble des répondants, sans qu'il soit possible d'évaluer si cette contrainte aurait aussi été perçue pour une autre technique. Concernant les étudiants, Torre et al. ${ }^{[11]}$ rapportent aussi que le temps requis pour construire la carte conceptuelle est une limite à son utilisation, qui pourrait peut-être être dépassée grâce à un entraînement. Les personnes habituées à la technique élaborent des cartes très vite. Il est reconnu que les étudiants exposés à des méthodes pédagogiques traditionnelles où l'apprentissage signifiant est peu valorisé développent des stratégies de mémorisation et de rappel efficaces mais souvent superficielles leur permettant d'avoir du succès dans leurs études en y accordant un minimum relatif de temps ${ }^{[14]}$. Par contraste, la décision d'exiger des étudiants qu'ils produisent des cartes conceptuelles s'inscrit très clairement dans une perspective pédagogique alternative, visant à favoriser le recours à des stratégies d'apprentissage en profondeur; au-delà des aspects techniques liés à la construction graphique de la carte, une telle démarche exige nécessairement un investissement en temps supérieur de la part des étudiants.

Un autre élément identifié comme un obstacle à l'utilisation des cartes conceptuelles construites par les étudiants est la détermination du niveau de détails à inclure. En effet, si la carte est dessinée à la main sur une feuille ou au tableau, le niveau de détails qu'il est possible d'inclure est habituellement défini par la grandeur de la feuille ou du tableau; lorsque l'on utilise un logiciel comme CmapTools, l'espace de travail virtuel possédant une dimension infinie, oblige l'enseignant à statuer au préalable sur le niveau de détail à inclure si l'on ne veut pas se retrouver avec une carte rendue illisible par le foisonnement des concepts qui y sont présentés.

\section{Conclusion}

Les résultats de cette étude exploratoire font apparaître que les pratiques pédagogiques actuellement associées à l'utilisation des cartes conceptuelles en sciences de la santé sont diversifiées. Ceci est potentiellement prometteur pour l'avenir, si l'on admet qu'il devient essentiel de promouvoir des stratégies d'apprentissage signifiant dans un contexte où les données factuelles croissent exponentiellement. Ce travail a également permis d'identifier à la fois des avantages consécutifs à l'utilisation des cartes conceptuelles mais aussi certains obstacles à leur utilisation. Comme l'ont souligné certains répondants, la carte conceptuelle n'est pas une fin en soi mais un moyen de favoriser un certain type d'activités cognitives cohérentes avec la perspective de l'apprentissage signifiant. À ce titre, il s'agit d'un outil utile à posséder dans son répertoire de moyens pédagogiques et à utiliser adéquatement en fonction des finalités à atteindre. 


\section{Contributions}

Annie Rochette, Marilou Bélisle, André Laflamme, Michèle Doucet et Monique Chaput ont contribué à la conception du protocole de recherche. André Laflamme a pris en charge le recueil des données. Annie Rochette et Barbara Fillion ont fait l'analyse des données avec la collaboration des autres auteurs. Annie Rochette et Barbara Fillion ont procédé à la rédaction du manuscrit et tous les autres auteurs l'ont révisé à multiples reprises.

\section{Remerciements}

Cette étude a été réalisée grâce à un fonds de recherche du Centre de recherche interdisciplinaire en réadaptation de Montréal (CRIR) et du site Centre de réadaptation Lucie-Bruneau (CRLB) octroyé à l'auteure principale. Annie Rochette est également détentrice d'une bourse de chercheur Junior 1 du Fonds de la Recherche en Santé du Québec (FRSQ).

\section{Approbation éthique}

Le protocole de recherche de cette étude a été approuvé par le Comité d'éthique de la recherche de la Faculté de médecine de l'Université de Montréal (CERFM; 07-\#282).

\section{Références}

1. Frank JR. (Ed). The CanMEDS 2005 physician competency framework. Better standards. Better physicians. Better care. Ottawa: The Royal College of Physicians and Surgeons of Canada, 2005 [Online] Disponible sur : http://crmcc.medical.org/ canmeds/CanMEDS2005/.

2. Association canadienne des ergothérapeutes. Profil de la pratique de l'ergothérapie au Canada. Ottawa : CAOT Publications ACE, 2007.
3. Tardif J. L'évaluation des compétences: Documenter le parcours de développement. Montréal (QC) : Chenelière Éducation, 2006.

4. Eitel F, Kanz KG, Hortig E, Tesche A. Do we face a fourth paradigm shift in medicine-algorithms in education? J Eval Clin Pract 2000;6:321-33.

5. Daley BJ, Torre DM. Concept maps in medical education: an analytical literature review. Med Educ 2010;44:440-8.

6. Ausubel DP. Educational Psychology: A Cognitive View. New York : Holt, Reinhart \& Winston 1968.

7. Marchand C, Ivernois J-F. Les cartes conceptuelles dans les formations en santé. Pédagogie Médicale 2004;5:230-40.

8. Kinchin I. If concept mapping is so helpful to learning biology, why aren't we all doing it? Int J Sci Educ 2001;23:1257-69.

9. Novak JD, Gowin DB. Learning How to Learn. New York : Cambridge University Press 1984.

10. Rochette A, Korner-Bitensky N, Thomas A. Changing clinicians' habits: Is this the hidden challenge to increasing best practices? Disabil Rehabil: 2009;31(21):1790-4.

11. Torre DM, Daley B, Stark-Schweitzer T, Siddartha S, Petkova J, Ziebert M. A qualitative evaluation of medical student learning with concept maps. Med Teach 2007;29:949-55.

12. Hilbert TS, Renkl A. Concept mapping as a follow-up strategy to learning from texts: what characterizes good and poor mappers? Instr Sci 2008;36:53-73.

13. Pudelko B, Basque J. Logiciels de construction de cartes de connaissances : des outils pour apprendre. 2005 [Online] Disponible sur : http://wwW.profetic.org/ dossiers/spip.php?rubrique108.

14. Novak JD. The Promise of New Ideas and New Technology for Improving Teaching and Learning. Cell Biology Education 2003;2:122-32.
Correspondance et offprints : Annie Rochette, École de Réadaptation, Université de Montréal C.P. 6128, Succursale Centre-Ville H3C 3J7 Montréal, Québec, Canada.

Mailto : annie.rochette@umontreal.ca 